\title{
DROP: A Data Reduction and Organization Paradigm and its Application in Image Analysis
}

\author{
Priscila T. M. Saito \\ Department of Computing, Federal University of Technology - Parana, Cornelio Procopio, Brazil \\ Advisors: Alexandre X. Falcão, Pedro J. de Rezende \\ Data and location of the PhD thesis defense: 28th April 2014, \\ Institute of Computing, University of Campinas, Campinas, Brazil
}

Received 28th February 2015; accepted 15th May 2015

\begin{abstract}
Recent technological advancements have provided large image datasets from distinctive domains (e.g., medicine, remote sensing, multimedia applications, among others) over the past two decades. It becomes an important challenge to analyze and organize these images effectively. In this context, automatic image annotation [5] has received much attention in research. Nevertheless, there is an inherent trade-off, wherein the human knowledge is indispensable for the success of the process and the human's time and effort are precious resources.

Considering that a great number of unlabeled images is available, it is beneficial to develop an effective approach to select a considerably lower number of the most informative samples to train a pattern classifier under human's supervision. Active learning techniques [1] have been investigated with this purpose. However, the majority of them very often ignores the need for interactive response times during the active learning process. They usually adopt a common paradigm (Figure 1a) which requires, at every learning iteration, the classification and/or organization of the entire dataset. This paradigm is impractical when considering large datasets.

The $\mathrm{PhD}$ research in [2] addressed these issues by proposing an effective and efficient Data Reduction and Organization Paradigm (DROP). In our paradigm (Figure 1b), the proposed active learning methods should be able to reduce and/or organize the large dataset such that sample selection does not require to reprocess it entirely at each learning iteration. Thus, the selection process becomes faster, since the improvement of the classifier at each iteration does not require rearranging all samples. Furthermore, a faster selection process is completed when the classifier chooses the desired number of samples.

The proposed paradigm aims to select unlabeled samples from all classes for user annotation at the first learning iteration and then the most informative (most difficult) samples for classification at the subsequent iterations. Being a paradigm, DROP can be implemented with different strategies for the reduction, organization and selection processes. In general, the adopted strategies [3] rely on graph-based clustering. From the clustering, we gain valuable information, since representative samples located at the center of the clusters (root samples) are more likely to cover all classes and are good candidates to be selected first for manual annotation.
\end{abstract}

Correspondence to: <psaito@utfpr.edu.br>

Recommended for acceptance by Jorge Bernal

DOI http://dx.doi.org/10.5565/rev/elcvia.727

ELCVIA ISSN:1577-5097

Published by Computer Vision Center / Universitat Autònoma de Barcelona, Barcelona, Spain 


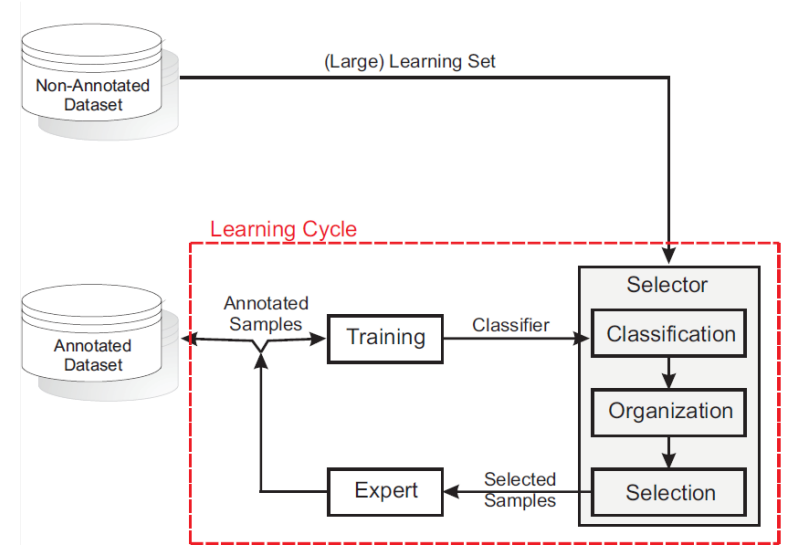

(a)

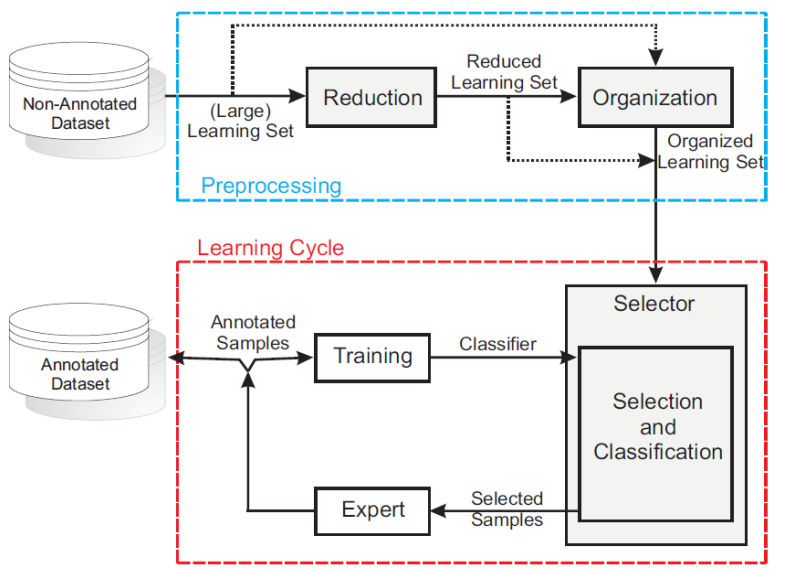

(b)

Figure 1: Pipelines for active learning: (a) traditional paradigm and (b) proposed one.

Moreover, samples in the same cluster are likely to have the same label. This assumption is used to accelerate active learning by reducing the number of annotating samples from the same cluster. Cluster roots, boundary between distinct cluster samples, and other specific criteria allow to select the most informative samples earlier for the training of the classifier.

Our active learning strategies iteratively seek to select the most informative samples based on the current knowledge of both human and classifier, at each iteration. The classifier actively participates of its learning process by classifying and supporting the selection of the samples. Since the selected samples are automatically labeled by the current classifier, the user has only to verify the assigned labels and annotate the misclassified ones. As the classifier improves throughout the iterations, the number of samples incorrectly classified is considerably reduced, indicating to the user when to stop the learning process. In this way, the human's time and effort are also increasingly lessened.

The proposed strategies were extensively assessed with different types of unsupervised and supervised classifiers by using datasets from distinct application domains, such as: image segmentation, forest cover type, handwritten digits, faces, cowhide, as well as image annotation from a real application for the diagnosis of parasites [4]. The experiments performed on these datasets show that the proposed approaches outperform the baseline ones, requiring only a few iterations to achieve high accuracy and with less human involvement.

\section{References}

[1] Ajay J. Joshi, Fatih Porikli, and Nikolaos P. Papanikolopoulos. Scalable active learning for multi-class image classification. IEEE Trans. on Pattern Analysis and Machine Intelligence, 34(11):2259-2273, 2012.

[2] Priscila T. M. Saito. Active learning with applications to the diagnosis of parasites. $\mathrm{PhD}$ Thesis, Institute of Computing, University of Campinas (IC-UNICAMP). Available: http://www.bibliotecadigital.unicamp.br/document/?code=000931972, 2014.

[3] Priscila T. M. Saito, Pedro J. de Rezende, Alexandre X. Falcão, Celso T. N. Suzuki, and Jancarlo F. Gomes. An active learning paradigm based on a priori data reduction and organization. Expert Systems with Applications, 41(14):6086-6097, 2014.

[4] Priscila T. M. Saito, Celso T. N. Suzuki, Jancarlo F. Gomes, Alexandre X. Falcão, and Pedro J. de Rezende. Robust active learning for the diagnosis of parasites. Pattern Recognition, 48:3572-3583, 2015.

[5] Dengsheng Zhang, Md. Monirul Islam, and Guojun Lu. A review on automatic image annotation techniques. Pattern Recognition, 45(1):346-362, 2012. 